PROCEEDINGS OF THE

AMERICAN MATHEMATICAL SOCIETY

Volume 133, Number 3, Pages 815-825

S 0002-9939(04)07601-4

Article electronically published on September 29, 2004

\title{
FRAMES, MODULAR FUNCTIONS FOR SHIFT-INVARIANT SUBSPACES AND FMRA WAVELET FRAMES
}

\author{
QING GU AND DEGUANG HAN
}

(Communicated by David R. Larson)

\begin{abstract}
We introduce the concept of the modular function for a shiftinvariant subspace that can be represented by normalized tight frame generators for the shift-invariant subspace and prove that it is independent of the selections of the frame generators for the subspace. We shall apply it to study the connections between the dimension functions of wavelet frames for any expansive integer matrix $A$ and the multiplicity functions for general multiresolution analysis (GMRA). Given a frame mutiresolution analysis (FMRA), we show that the standard construction formula for orthonormal multiresolution analysis wavelets does not yield wavelet frames unless the underlying FMRA is an MRA. A modified explicit construction formula for FMRA wavelet frames is given in terms of the frame scaling functions and the low-pass filters.
\end{abstract}

\section{Preliminaries}

A frame for a separable Hilbert space $H$ is a sequence of vectors $\left\{f_{j}\right\}$ in $H$ such that there exist constants $C_{1}, C_{2}>0$ such that

$$
C_{1}\|f\|_{2}^{2} \leq \sum_{j}\left|\left\langle f, f_{j}\right\rangle\right|^{2} \leq C_{2}\|f\|_{2}^{2}
$$

holds for all $f \in H$. If $C_{1}=C_{2}=1$, we say that $\left\{f_{j}\right\}$ is a normalized tight frame.

For a $d \times d$ real expansive matrix $A$ (i.e., all the eigenvalues of $A$ are required to have absolute values greater than 1), an A-dilation (orthogonal) wavelet is a single function $\psi \in L^{2}\left(\mathbb{R}^{d}\right)$ with the property that

$$
\psi_{m, \ell}^{A}(t):=\left\{|\operatorname{det} A|^{\frac{m}{2}} \psi\left(A^{m} t-\ell\right): m \in \mathbb{Z}, \ell \in \mathbb{Z}^{d}\right\}
$$

is an orthonormal basis for $L^{2}\left(\mathbb{R}^{d}\right)$. More generally, $\psi$ will be called a normalized tight A-dilation wavelet frame if $\left\{\psi_{m, \ell}^{A}\right\}$ forms a normalized tight frame for $L^{2}\left(\mathbb{R}^{d}\right)$. In what follows we will use the term "wavelet frame" to denote the normalized tight ones.

The dilation operator $\delta_{A}$ and the translation operator $T_{\ell}$ are defined by:

$$
\left(\delta_{A} f\right)(t)=|\operatorname{det} A|^{1 / 2} f(A t), \quad\left(T_{\ell} f\right)(t)=f(t-\ell)
$$

Received by the editors February 25, 2002 and, in revised form, November 11, 2003.

2000 Mathematics Subject Classification. Primary 42C15, 47B38.

Key words and phrases. Wavelet, wavelet frame, frame multiresolution analysis, shift-invariant subspace, dimension function.

This paper is a revised version based on an earlier circulated preprint: "Translation invariant subspaces and general multiresolution analysis", 1999.

(C)2004 American Mathematical Society 
for all $f \in L^{2}\left(\mathbb{R}^{d}\right)$. A closed subspace $M$ of $L^{2}\left(\mathbb{R}^{d}\right)$ is called shift-invariant if $T_{\ell} M=M$ for any $\ell \in \mathbb{Z}^{d}$. The Fourier transform of $f \in L^{1}\left(\mathbb{R}^{d}\right) \cap L^{2}\left(\mathbb{R}^{d}\right)$ is defined by

$$
(\mathcal{F} f)(\xi):=\frac{1}{(2 \pi)^{d / 2}} \int_{\mathbb{R}^{d}} e^{-i\langle\xi, t\rangle} f(t) d \mu
$$

where $\mu$ is the Lebesgue measure on $\mathbb{R}^{d}$. Here we include a factor $\frac{1}{(2 \pi)^{d / 2}}$ in the definition of Fourier transform to guarantee that $\mathcal{F}$ preserves the inner product. Therefore, $\mathcal{F}$ can be extended to a unitary operator, still denoted by $\mathcal{F}$, on $L^{2}\left(\mathbb{R}^{d}\right)$. We will write $\hat{f}:=\mathcal{F}(f)$ and $\widehat{U}=\mathcal{F} U \mathcal{F}^{-1}$ for any $f \in L^{2}\left(\mathbb{R}^{d}\right)$ and any bounded operator $U$ on $L^{2}\left(\mathbb{R}^{d}\right)$.

A general multiresolution analysis (cf. $[\mathrm{Ba}],[\mathrm{BMM}],[\mathrm{BL},[\mathrm{Pa}]$ ) associated with a $d \times d$ expansive real matrix $A$ is a sequence $\left\{V_{j}: j \in \mathbb{Z}\right\}$ of closed subspaces of $L^{2}\left(\mathbb{R}^{d}\right)$ satisfying

(1) $V_{j} \subset V_{j+1}, j \in \mathbb{Z}$,

(2) $\bigcap_{j \in \mathbb{Z}} V_{j}=\{0\}, \overline{\bigcup_{j \in \mathbb{Z}} V_{j}}=L^{2}\left(\mathbb{R}^{d}\right)$,

(3) $f \in V_{j}$ if and only if $\delta_{A} f \in V_{j+1}, j \in \mathbb{Z}$,

(4) $V_{0}$ is invariant under the translation group $\left\{T_{\ell}: \ell \in \mathbb{Z}^{d}\right\}$.

If we require that $V_{0}$ contains a scaling function whose translates form an orthonormal basis for $V_{0}$, then $\left(V_{j}\right)$ is called a multiresolution analysis (MRA). Similarly, $\left(V_{j}\right)$ is called a frame multiresolution analysis (FMRA) if $V_{0}$ contains a frame scaling function $\phi$ whose translates form a normalized tight frame for $V_{0}$. Given a scaling function for an MRA, there is a standard way of constructing a wavelet in $V_{1} \ominus V_{0}$ (which is called an MRA wavelet, $\mathrm{Ma}$ ). However, the construction of FMRA wavelet frames is a more complicated issue. The purpose of this paper is twofold: (1) Give an explicit construction formula (Theorem 3.4) for FMRA wavelet frames, and (2) introduce the concept of the modular function for a shift-invariant subspace that can be regarded as a refinement of the multiplicity function (Theorem 2.2) and use it to explore the connections between the dimension function of a GMRA and the multiplicity function of the scaling space $V_{0}$.

The following theorem and its corollary follow from the consistency equation of GMRA in BMM] and will be used later.

Theorem 1.1. Let $\left(V_{j}\right)$ be a GMRA with a multi-frame scaling set $\left\{\phi_{\lambda}: \lambda \in \Lambda\right\}$, and let $A \mathbb{Z}^{d}, A \mathbb{Z}^{d}+\ell_{1}, \ldots, A \mathbb{Z}^{d}+\ell_{k}$ be a basis of the group $\mathbb{Z}^{d} / A \mathbb{Z}^{d}\left(\right.$ let $\ell_{0}=0$ ). Then there exists a wavelet frame $\psi \in W_{0}=V_{1} \ominus V_{0}$ if and only if

$$
\sum_{\lambda \in \Lambda} \sum_{i=0}^{k} \sum_{\ell \in \mathbb{Z}^{d}}\left|\hat{\phi}_{\lambda}\left(\left(A^{t}\right)^{-1}\left(\xi+2 \ell_{i} \pi\right)+2 \ell \pi\right)\right|^{2}=\sum_{\lambda \in \Lambda} \sum_{\ell \in \mathbb{Z}^{d}}\left|\hat{\phi}_{\lambda}(\xi+2 \ell \pi)\right|^{2}+\frac{1}{(2 \pi)^{d}} \chi_{E}
$$

for some measurable set $E$. In particular, if $\left(V_{j}\right)$ is an FMRA with a frame scaling function $\phi$, then there is a (orthogonal) wavelet in $W_{0}$ if and only if

$$
\sum_{i=0}^{k} \sum_{\ell \in \mathbb{Z}^{d}}\left|\hat{\phi}\left(\left(A^{t}\right)^{-1}\left(\xi+2 \ell_{i} \pi\right)+2 \ell \pi\right)\right|^{2}=\sum_{\ell \in \mathbb{Z}^{d}}|\hat{\phi}(\xi+2 \ell \pi)|^{2}+\frac{1}{(2 \pi)^{d}},
$$

where $A^{t}$ is the transpose of $A$.

The set $\left\{\ell_{i}\right\}$ in Theorem 1.1 is called a complete digit set for $A$. For any function $f \in L^{2}\left(\mathbb{R}^{d}\right)$, we define $\Phi_{f}(\xi)=\sum_{\ell \in \mathbb{Z}^{d}}|\hat{f}(\xi+2 \ell \pi)|^{2}$. Clearly $\operatorname{supp}\left(\Phi_{f}\right)=$ $\bigcup_{\ell \in \mathbb{Z}}(\operatorname{supp}(\hat{f})+2 \ell \pi)$. 
Corollary 1.2. Let $\left\{\ell_{0}, \ell_{1}, \ldots, \ell_{k}\right\}\left(\ell_{0}=0\right)$ be a complete digit set for A. Assume that $\left(V_{j}\right)$ is an FMRA such that $V_{0}=\overline{\operatorname{span}}\left\{T_{\ell} f: \ell \in \mathbb{Z}^{d}\right\}$. Let $F=\operatorname{supp}\left(\Phi_{f}\right)$ and $F_{i}=A^{t} F-2 \ell_{i} \pi$. Then $W_{0}$ is generated by a wavelet frame if and only if the following are satisfied:

(i) $F \subseteq \bigcup_{i=0}^{k} F_{i}$,

(ii) $F_{i} \cap F_{j} \subseteq F$ for $i \neq j$,

(iii) $F_{i} \cap F_{j} \cap F_{m}$ has measure zero for all different $i, j, m$.

Moreover, $W_{0}$ is generated by a wavelet if and only if the following are satisfied:

$\left(i^{\prime}\right) \bigcup_{i=0}^{k} F_{i}=\mathbb{R}^{d}$

(ii') $F=\bigcup_{i>j}\left(F_{i} \cap F_{j}\right)$,

(iii) $F_{i} \cap F_{j} \cap F_{m}$ has measure zero for all different $i, j, m$.

Proof. Let $\phi \in V_{0}$ be such that $\left\{T_{\ell} \phi: \ell \in \mathbb{Z}^{d}\right\}$ is a normalized tight frame for $V_{0}$. Then

$$
F=\operatorname{supp}\left(\Phi_{f}\right)=\operatorname{supp}\left(\Phi_{\phi}\right),
$$

and (1.1) becomes the condition that $\left(\sum_{i=0}^{k} \chi_{A^{t} F-2 \ell_{i} \pi}\right)-\chi_{F}=\chi_{E}$, which is clearly equivalent to $(i),($ ii $)$ and $($ iii $)$.

The last statement follows from the fact that $\left(i^{\prime}\right),\left(i i^{\prime \prime}\right)$ and $(i i i)$ are satisfied if and only if $\left(\sum_{i=0}^{k} \chi_{A^{t} F-2 \ell_{i} \pi}\right)-\chi_{F}=1$.

Remark 1.3. Keep all the notation in Corollary 1.2 and assume that $\left(V_{j}\right)$ is associated with a frame wavelet $\psi$. Then

$$
\sum_{\ell \in \mathbb{Z}^{d}}|\hat{\psi}(\xi+2 \ell \pi)|^{2}=\frac{1}{(2 \pi)^{d}} \chi_{E},
$$

with $E=\left(E_{1} \cup E_{2}\right)$, where $E_{1}=\bigcup_{i>j}\left(F_{i} \cap F_{j}\right)$ and $E_{2}=\left(\bigcup_{i=0}^{k}\left(F_{i} \backslash L_{i}\right)\right) \backslash F$ and $L_{i}=\bigcup_{j \neq i} F_{j}$.

The following is well known (cf. RS1, and also GHa for a more general version for any group) and will be needed in the rest of paper:

Lemma 1.4. Let $M$ be a shift-invariant subspace. Then there exists $\left\{\psi_{\lambda}: \lambda \in\right.$ $\Lambda\} \subset M$ such that $\left\{T_{\ell} \psi_{\lambda}: \ell \in \mathbb{Z}^{d}, \lambda \in \Lambda\right\}$ is a normalized tight frame for $M$.

\section{Tight FRAMES AND MUlTiPliCITY FUnCTIONS}

Let $\psi \in L^{2}(\mathbb{R})$ be a dyadic (dilation by 2 ) orthonormal wavelet. The dimension function of $\psi$ is defined by

$$
D_{\psi}(\xi)=\sum_{j \geq 1} \sum_{\ell \in \mathbb{Z}} \mid \hat{\psi}\left(\left.2^{j}(\xi+2 \ell \pi)\right|^{2} .\right.
$$

The importance of dimension functions for orthonormal wavelets was discovered by Lemarié [Le1, Le2] and Auscher [Aus. One of the applications is to use dimension functions to classify orthonormal wavelets. In particular, it is well known that a wavelet is an MRA wavelet if and only if $(2 \pi) D_{\psi}(\xi)=1$, a.e. $\xi \in \mathbb{R}$. The reason behind this is that the dimension function of $\psi$ is the multiplicity function for the scaling space $V_{0}$ associated with $\psi$ (cf. We for the definition of multiplicity functions for shift-invariant subspaces). Although some evidence shows that this must be true for any (multi) wavelet frames, it has not been proved yet (cf. [Ba], [BRS]). The purpose of this section is to provide a result concerning the modules for normalized tight frame generators, which seems first to be observed and from 
which we will see a more transparent connection between dimension and multiplicity functions.

For any collection $\Psi=\left\{\psi_{\lambda}: \lambda \in \Lambda\right\}$ of functions in $L^{2}\left(\mathbb{R}^{d}\right)$, let

$$
D_{\Psi}(\xi)=\sum_{\lambda \in \Lambda} \sum_{j \geq 1} \sum_{\ell \in \mathbb{Z}^{d}}\left|\hat{\psi}_{\lambda}\left(B^{j}(\xi+2 \ell \pi)\right)\right|^{2},
$$

where $B=A^{t}$ is the transpose of $A$.

Lemma 2.1. Let $M$ be a shift-invariant subspace. Assume that both $\left\{T_{\ell} \psi_{\lambda}: \ell \in\right.$ $\left.\mathbb{Z}^{d}, \lambda \in \Lambda\right\}$ and $\left\{T_{\ell} \phi_{\gamma}: \ell \in \mathbb{Z}^{d}, \gamma \in \Gamma\right\}$ are normalized tight frames for $M$. Then

$$
\sum_{\lambda \in \Lambda} \sum_{\ell \in \mathbb{Z}^{d}}\left|\hat{\psi}_{\lambda}(\xi+2 \ell \pi)\right|^{2}=\sum_{\gamma \in \Gamma} \sum_{\ell \in \mathbb{Z}^{d}}\left|\hat{\phi}_{\gamma}(\xi+2 \ell \pi)\right|^{2} .
$$

In particular, the above function is equal to the multiplicity function $m_{M}(\xi)$.

Proof. Let $F \subset \mathbb{T}^{d}$ be any measurable subset, and let $E=\bigcup_{\ell \in \mathbb{Z}}(F+2 \ell \pi)$. Then $\chi_{E}$ is a $2 \pi$-periodic function and $\chi_{E} \hat{M} \subset \hat{M}$ since $\hat{M}$ is invariant under multiplication by $e^{2 \pi i\langle\ell, \xi\rangle}$ for all $\ell \in \mathbb{Z}^{d}$. This would imply that both $\left\{\frac{1}{(2 \pi)^{d}} \hat{T}_{\ell} \chi_{E} \hat{\psi}_{\lambda}: \ell \in \mathbb{Z}^{d}, \lambda \in\right.$ $\Lambda\}$ and $\left\{\frac{1}{(2 \pi)^{d}} \hat{T}_{\ell} \chi_{E} \hat{\phi}_{\gamma}: \ell \in \mathbb{Z}^{d}, \gamma \in \Gamma\right\}$ are normalized tight frames for $\chi_{E} \hat{M}$. Thus

$$
\begin{aligned}
\int_{F} \sum_{\lambda \in \Lambda} \sum_{\ell \in \mathbb{Z}^{d}}\left|\hat{\psi}_{\lambda}(\xi+2 \ell \pi)\right|^{2} & =\sum_{\lambda \in \Lambda}\left\|\chi_{E} \hat{\psi}_{\lambda}\right\|^{2} \\
& =\sum_{\lambda \in \Lambda} \sum_{\gamma \in \Gamma} \sum_{\ell \in \mathbb{Z}^{d}}\left|\left\langle\chi_{E} \hat{\psi}_{\lambda}, \frac{1}{(2 \pi)^{d}} \hat{T}_{\ell} \chi_{E} \hat{\phi}_{\gamma}\right\rangle\right|^{2} \\
& =\sum_{\gamma \in \Gamma} \sum_{\lambda \in \Lambda} \sum_{\ell \in \mathbb{Z}^{d}}\left|\left\langle\frac{1}{(2 \pi)^{d}} \hat{T_{-\ell} \chi_{E}} \hat{\psi}_{\lambda}, \chi_{E} \hat{\phi}_{\gamma}\right\rangle\right|^{2} \\
& =\sum_{\gamma \in \Gamma}\left\|\chi_{E} \hat{\phi}_{\gamma}\right\|^{2} \\
& =\int_{F} \sum_{\gamma \in \Gamma} \sum_{\ell \in \mathbb{Z}^{d}}\left|\hat{\phi}_{\gamma}(\xi+2 \ell \pi)\right|^{2}
\end{aligned}
$$

Since $F$ is an arbitrary measurable set of $\mathbb{T}^{d}$, it follows that

$$
\sum_{\lambda \in \Lambda} \sum_{\ell \in \mathbb{Z}^{d}}\left|\hat{\psi}_{\lambda}(\xi+2 \ell \pi)\right|^{2}=\sum_{\gamma \in \Gamma} \sum_{\ell \in \mathbb{Z}^{d}}\left|\hat{\phi}_{\gamma}(\xi+2 \ell \pi)\right|^{2} .
$$

For the last statement, we can decompose $M$ into an orthogonal direct sum of cyclic shift-invariant subspaces $S_{i}$. Let $\phi_{i} \in S_{i}$ be such that its translations generate a normalized tight frame. Then it is obvious that the multiplicity function $m_{i}$ is equal to $\sum_{\ell \in \mathbb{Z}^{d}}\left|\hat{\phi}_{i}(\xi+2 \ell \pi)\right|^{2}$. Hence $m_{M}(\xi)=\sum_{i \in \mathcal{I}} \sum_{\ell \in \mathbb{Z}^{d}}\left|\hat{\phi}_{i}(\xi+2 \ell \pi)\right|^{2}$. Thus, by what we have proved, $m_{M}(\xi)=\sum_{\lambda \in \Lambda} \sum_{\ell \in \mathbb{Z}^{d}}\left|\hat{\psi}_{\lambda}(\xi+2 \ell \pi)\right|^{2}$ for any normalized tight frame $\left\{T_{\ell} \psi_{\lambda}: \ell \in \mathbb{Z}^{d}, \lambda \in \Lambda\right\}$ of $M$.

In the case that the multiplicity function is finite we can prove the following much stronger result:

Theorem 2.2. Let $M$ be a shift-invariant subspace such that its multiplicity function $m_{M}(\xi)$ is finite almost everywhere. If both $\left\{T_{\ell} \psi_{\lambda}: \lambda \in \Lambda \ell \in \mathbb{Z}^{d}\right\}$ and 
$\left\{T_{\ell} \phi_{\gamma}: \gamma \in \Gamma, \ell \in \mathbb{Z}^{d}\right\}$ are normalized tight frames for $M$, then

$$
\sum_{\lambda \in \Lambda}\left|\hat{\psi}_{\lambda}(\xi)\right|^{2}=\sum_{\gamma \in \Gamma}\left|\hat{\phi}_{\gamma}(\xi)\right|^{2}, \text { a.e. }
$$

We call the function represented by (2.1) the modular function of $M$. Clearly the multiplicity function is the periodization of the modular function.

Case I. $\sum_{\lambda \in \Lambda}\left\|\psi_{\lambda}\right\|^{2}<\infty$ (equivalently, $m_{M}(\xi)$ is integrable on $\mathbb{T}^{d}$ ).

Let $E$ be any measurable subset of $\mathbb{R}^{d}$.

$$
\begin{aligned}
\sum_{\lambda \in \Lambda}\left\|\chi_{E} \hat{\psi}_{\lambda}\right\|^{2} & =\sum_{\lambda \in \Lambda}\left\langle\chi_{E} \hat{\psi}_{\lambda}, \hat{\psi}_{\lambda}\right\rangle \\
& =\frac{1}{2 \pi^{d}} \sum_{\lambda \in \Lambda} \sum_{\gamma \in \Gamma}\left\langle\chi_{E} \hat{\psi}_{\lambda}, \sum_{\ell \in \mathbb{Z}^{d}}\left\langle\hat{\psi}_{\lambda}, \hat{T}_{\ell} \hat{\phi}_{\gamma}\right\rangle \hat{T}_{\ell} \hat{\phi}_{\gamma}\right\rangle \\
& =\frac{1}{2 \pi^{d}} \sum_{\lambda \in \Lambda} \sum_{\gamma \in \Gamma} \sum_{\ell \in \mathbb{Z}^{d}}\left\langle\chi_{E} \hat{\psi}_{\lambda},\left\langle\hat{\psi}_{\lambda}, \hat{T}_{\ell} \hat{\phi}_{\gamma}\right\rangle \hat{T}_{\ell} \hat{\phi}_{\gamma}\right\rangle \\
& =\frac{1}{2 \pi^{d}} \sum_{\gamma \in \Gamma} \sum_{\lambda \in \Lambda} \sum_{\ell \in \mathbb{Z}^{d}}\left\langle\hat{\phi}_{\gamma}, \hat{T}_{-\ell} \hat{\psi}_{\lambda}\right\rangle\left\langle\hat{T}_{-\ell} \chi_{E} \hat{\psi}_{\lambda}, \hat{\phi}_{\gamma}\right\rangle \\
& =\frac{1}{2 \pi^{d}} \sum_{\gamma \in \Gamma} \sum_{\lambda \in \Lambda} \sum_{\ell \in \mathbb{Z}^{d}}\left\langle\hat{\phi}_{\gamma}, \hat{T}_{-\ell} \hat{\psi}_{\lambda}\right\rangle\left\langle\hat{T}_{-\ell} \hat{\psi}_{\lambda}, \chi_{E} \hat{\phi}_{\gamma}\right\rangle \\
& =\sum_{\gamma \in \Gamma}\left\langle\hat{\phi}_{\gamma}, \chi_{E} \hat{\phi}_{\gamma}\right\rangle \\
& =\sum_{\gamma \in \Gamma}\left\|\chi_{E} \hat{\phi}_{\gamma}\right\|^{2},
\end{aligned}
$$

where in the fourth equality we could interchange the summations because of the assumption $\sum_{\lambda \in \Lambda}\left\|\psi_{\lambda}\right\|^{2}<\infty$. Since $E$ is arbitrary, it follows that

$$
\sum_{\lambda \in \Lambda}\left|\hat{\psi}_{\lambda}(\xi)\right|^{2}=\sum_{\gamma \in \Gamma}\left|\hat{\phi}_{\gamma}(\xi)\right|^{2}, \text { a.e. }
$$

Case II. The general case.

Define $F_{n}=\left\{\xi \in \mathbb{T}^{d}: m_{M}(\xi) \leq n\right\}$, and let $E_{n}=\bigcup_{\ell \in \mathbb{Z}^{d}}\left(F_{n}+2 \ell \pi\right)$. Then, by assumption, $\bigcup_{n=1}^{\infty} F_{n}=\mathbb{T}^{d}$ and $\bigcup_{n=1}^{\infty} E_{n}=\mathbb{R}^{d}$ modulo null sets. As discussed in the proof of Lemma 2.1, both $\left\{\frac{1}{(2 \pi)^{d}} \hat{T}_{\ell} \chi_{E_{n}} \hat{\psi}_{\lambda}: \ell \in \mathbb{Z}^{d}, \lambda \in \Lambda\right\}$ and $\left\{\frac{1}{(2 \pi)^{d}} \hat{T}_{\ell} \chi_{E_{n}} \hat{\phi}_{\gamma}\right.$ : $\left.\ell \in \mathbb{Z}^{d}, \gamma \in \Gamma\right\}$ are normalized tight frames for $\chi_{E_{n}} \hat{M}$. Moreover,

$$
\sum_{\lambda}\left\|\chi_{E_{n}} \hat{\psi}_{\lambda}\right\|^{2} \leq n(2 \pi)^{d}<\infty .
$$

So by applying Case I to the shift-invariant subspace that is the image of the inverse Fourier transform of $\chi_{E_{n}} \hat{M}$, we get

$$
\sum_{\lambda \in \Lambda}\left|\chi_{E_{n}}(\xi) \hat{\psi}_{\lambda}(\xi)\right|^{2}=\sum_{\gamma \in \Gamma}\left|\chi_{E_{n}}(\xi) \hat{\phi}_{\gamma}(\xi)\right|^{2} \text {, a.e. }
$$

Hence

$$
\sum_{\lambda \in \Lambda}\left|\hat{\psi}_{\lambda}(\xi)\right|^{2}=\sum_{\gamma \in \Gamma}\left|\hat{\phi}_{\gamma}(\xi)\right|^{2}, \quad \text { a.e. }
$$

since $\bigcup_{n=1}^{\infty} E_{n}=\mathbb{R}^{d}$. 
Corollary 2.3. Let $M$ be a shift-invariant subspace such that its multiplicity function $m_{M}(\xi)$ is finite almost everywhere. If both $\Psi=\left\{\psi_{\lambda}: \lambda \in \Lambda\right\}$ and $\Phi=\left\{\phi_{\gamma}\right.$ : $\gamma \in \Gamma\}$ generate normalized tight frames for $M$, then

$$
D_{\Psi}(\xi)=D_{\Phi}(\xi) \text {. }
$$

Proof. By Theorem 2.2, we have that for any $j \in \mathbb{Z}$,

$$
\sum_{\lambda \in \Lambda} \sum_{\ell \in \mathbb{Z}^{d}}\left|\hat{\psi}_{\lambda}\left(B^{j}(\xi+2 \ell \pi)\right)\right|^{2}=\sum_{\gamma \in \Gamma} \sum_{\ell \in \mathbb{Z}^{d}}\left|\hat{\phi}_{\gamma}\left(B^{j}(\xi+2 \ell \pi)\right)\right|^{2} .
$$

Summing over $j>0$ on both sides, we get $D_{\Psi}(\xi)=D_{\Phi}(\xi)$.

We conjecture that the condition concerning the multiplicity function of $M$ in Theorem 2.2 and Corollary 2.3 can be dropped.

Proposition 2.4. Let $V_{0}$ and $M$ be shift-invariant subspaces such that $M \perp V_{0}$ and $\delta_{A} V_{0}=V_{0} \oplus M$. If $m_{V_{0}} \in L^{1}(\Omega)$, then

$$
m_{V_{0}}(\xi)=D_{\Psi}(\xi)
$$

where $\Omega=[0,2 \pi)^{d}$ and $\Psi:=\left\{\psi_{\lambda}: \lambda \in \Lambda\right\} \subset M$ such that its translates form a normalized tight frame for $M$.

Proof. Let $\left\{T_{\ell} \phi_{\gamma}: \gamma \in \Gamma \ell \in \mathbb{Z}^{d}\right\}$ be a normalized tight frame for $V_{0}$. By the assumption we have $\sum_{\gamma \in \Gamma}\left\|\phi_{\gamma}\right\|^{2}<\infty$.

Let $\Psi=\left\{\psi_{\lambda}: \lambda \in \Lambda\right\}$ generate a normalized tight frame for $M$. In this case $m_{M}(\xi)<\infty$ (in fact $\left.\sum_{\lambda}\left\|\psi_{\lambda}\right\|^{2}<\infty\right)$. Let $q=|\operatorname{det} A|$ and $\left\{\ell_{0}, \ldots, \ell_{q-1}\right\}$ be a full digit set for $B$, i.e. $\left\{A \mathbb{Z}^{d}+\ell_{k}: 1 \leq k \leq d-1\right\}$ is a basis for the quotient group $\mathbb{Z}^{d} / A \mathbb{Z}^{d}$. Then both

$$
\left\{T_{\ell} \phi_{\gamma}: \gamma \in \Gamma \ell \in \mathbb{Z}^{d}\right\} \cup\left\{T_{\ell} \psi_{\lambda}: \lambda \in \Lambda, \ell \in \mathbb{Z}^{d}\right\}
$$

and

$$
\left\{T_{\ell} \delta_{A} T_{\ell_{k}} \phi_{\gamma}: \gamma \in \Gamma \ell \in \mathbb{Z}^{d}, 1 \leq k \leq q-1\right\}
$$

are normalized tight frames for $V_{0} \oplus M$. Let $g_{k, \gamma}=\delta_{A} T_{\ell_{k}} \phi_{\gamma}$. Then $\hat{g}_{k, \gamma}(\xi)=$ $\frac{1}{|\operatorname{det} A|^{1 / 2}} e^{i\left\langle B^{-1} \xi \ell_{k}\right\rangle} \hat{\phi}_{\gamma}\left(B^{-1} \xi\right)$. Applying Theorem 2.2 to $V_{0} \oplus M$ and these two normalized tight frames, we have

$$
\begin{aligned}
\sum_{j=1}^{\infty} \sum_{\gamma \in \Gamma} \sum_{\ell \in \mathbb{Z}^{d}}\left|\hat{\phi}_{\gamma}\left(B^{j}(\xi+2 \ell \pi)\right)\right|^{2}+\sum_{j=1}^{\infty} \sum_{\lambda} \sum_{\ell \in \mathbb{Z}^{d}}\left|\hat{\psi}_{\lambda}\left(B^{j}(\xi+2 \ell \pi)\right)\right|^{2} \\
=\sum_{k=0}^{q-1} \sum_{j=1}^{\infty} \sum_{\gamma \in \Gamma} \sum_{\ell \in \mathbb{Z}^{d}} \mid\left(\left.\hat{g}_{k, \gamma}\left(B^{j}(\xi+2 \ell \pi)\right)\right|^{2}\right. \\
=(q / q) \sum_{j=1}^{\infty} \sum_{\gamma \in \Gamma} \sum_{\ell \in \mathbb{Z}^{d}}\left|\hat{\phi}_{\gamma}\left(B^{j-1}(\xi+2 \ell \pi)\right)\right|^{2} \\
=\sum_{j=1}^{\infty} \sum_{\gamma \in \Gamma} \sum_{\ell \in \mathbb{Z}^{d}}\left|\hat{\phi}_{\gamma}\left(B^{j}(\xi+2 \ell \pi)\right)\right|^{2}+\left.\sum_{\gamma \in \Gamma} \sum_{\ell \in \mathbb{Z}^{d}} \hat{\phi}_{\gamma}(\xi+2 \ell \pi)\right|^{2},
\end{aligned}
$$

that is,

$$
D_{\Phi}(\xi)+D_{\Psi}(\xi)=D_{\Phi}(\xi)+\sum_{\gamma \in \Gamma} \sum_{\ell \in \mathbb{Z}^{d}}\left|\hat{\phi}_{\gamma}(\xi+2 \ell \pi)\right|^{2}
$$


From the assumption, $D_{\Phi}(\xi)<\infty$, a.e., which implies that

$$
m_{V_{0}}(\xi)=\sum_{\gamma \in \Gamma} \sum_{\ell \in \mathbb{Z}^{d}}\left|\hat{\phi}_{\gamma}(\xi+2 \ell \pi)\right|^{2}=D_{\psi}(\xi) \text {, a.e. }
$$

Applying Proposition 2.4 to wavelet frames, we have

Corollary 2.5. Assume that $\left(V_{j}\right)$ is a GMRA and $\Phi=\left\{\psi_{\lambda}: \lambda \in \Lambda\right\}$ generates a normalized tight frame for $V_{1} \ominus V_{0}$. If $m_{V_{0}}(\xi) \in L^{1}(\Omega)$, then $m_{V_{0}}(\xi)=D_{\Psi}(\xi)$.

Remark. Various forms of Proposition 2.4 have appeared in different contexts in the literature. In particular, in a recent paper RS2 Ron and Shen also proved this result. (We thank the referee for bringing our attention to Ron and Shen's new paper.) However, our treatment here seems more transparent and elementary. Moreover, Theorem 2.2 is a much stronger statement about the multiplicity functions for shift-invariant subspaces, and it seems to be new in the literature.

\section{The Construction of FMRA WAVElet frames}

In this section we discuss the constructions of FMRA wavelet frames. We focus on the case $A=2$ and $d=1$. It is well known that if $\phi$ is a scaling function for an MRA, then there is a unique $2 \pi$-periodic function $m_{0} \in L^{2}(\mathbb{T})$ satisfying

$$
\hat{\phi}(\xi)=m_{0}(\xi / 2) \hat{\phi}(\xi / 2),
$$

and $\psi$ defined by

$$
\hat{\psi}(\xi)=e^{i \xi / 2} \overline{m_{0}(\xi / 2+\pi)} \hat{\phi}(\xi / 2)
$$

is a wavelet. In the frame scaling function case, $m_{0}$ is not necessarily unique. We will first prove that for any $2 \pi$-periodic function $m_{0} \in L^{2}(\mathbb{T})$ satisfying (3.1), then $\psi$ defined by (3.2) will never be a wavelet frame unless the associated FMRA is an MRA. To prove this we need the following Lemma 3.1. For any two sets $E$ and $F$, we write $E \Delta F=(E \backslash F) \cup(F \backslash E)$.

Lemma 3.1. Let $\left(V_{j}\right)$ be an FMRA with a frame scaling function $\phi$, and let $m_{0}$ be as in (3.1). Write $F=\bigcup_{k \in \mathbb{Z}}(\operatorname{supp}(\phi)+2 k \pi)$. Assume that $W_{0}$ is generated by a wavelet frame. Then

$$
\left|m_{0}(\xi)\right|^{2}+\left|m_{0}(\xi+\pi)\right|^{2}= \begin{cases}1, & \xi \in F \cap(F-\pi), \\ 0, & \xi \in(F \Delta(F-\pi)) \backslash(F / 2) .\end{cases}
$$

Proof. Using the connection between $m_{0}$ and $\hat{\phi}$, we have

$$
\begin{aligned}
\sum_{k \in \mathbb{Z}}|\hat{\phi}(\xi+2 k \pi)|^{2} & =\left|m_{0}(\xi / 2)\right|^{2} \sum_{k \in \mathbb{Z}}|\hat{\phi}(\xi / 2+2 k \pi)|^{2} \\
& +\left|m_{0}(\xi / 2+\pi)\right|^{2} \sum_{k \in \mathbb{Z}}|\hat{\phi}(\xi / 2+\pi+2 k \pi)|^{2} .
\end{aligned}
$$

This implies that

$$
\chi_{F}(\xi)=\left|m_{0}(\xi / 2)\right|^{2} \chi_{2 F}(\xi)+\left|m_{0}(\xi / 2+\pi)\right|^{2} \chi_{2 F-2 \pi}(\xi),
$$

i.e.,

$$
\chi_{F / 2}(\xi)=\left|m_{0}(\xi)\right|^{2} \chi_{F}(\xi)+\left|m_{0}(\xi+\pi)\right|^{2} \chi_{F-\pi}(\xi) .
$$


Now let $\xi \in F \cap(F-\pi)$. By Corollary 1.2, $F \cap(F-\pi) \subset F / 2$. Thus $\left|m_{0}(\xi)\right|^{2}+$ $\left|m_{0}(\xi+\pi)\right|^{2}=1$ by the above identity. Similarly

$$
\left|m_{0}(\xi)\right|^{2}+\left|m_{0}(\xi+\pi)\right|^{2}=0
$$

when $\xi \in(F \Delta(F-\pi)) \backslash(F / 2)$.

Proposition 3.2. Let $\left(V_{j}\right)$ be an FMRA with a frame scaling function $\phi$ and $\psi$ be defined by (3.2). Then the following are equivalent:

(i) $\left(V_{j}\right)$ is an $M R A$,

(ii) $\psi \in W_{0}$ is a normalized tight frame wavelet.

Proof. We only need to verify the implication of $(i i) \Rightarrow(i)$. Let $F=\operatorname{supp}\left(\Phi_{\phi}\right)$. Since the frame scaling function $\phi$ is an (orthogonal) scaling function if and only if $F=\mathbb{R}$ (modulo a null set), it suffices to check that $F=\mathbb{R}$ (modulo a null set). By (3.2), we can obtain the following identity:

$$
(2 \pi) \sum_{k \in \mathbb{Z}}|\hat{\psi}(\xi+2 k \pi)|^{2}=\left|m_{0}(\xi / 2+\pi)\right|^{2} \chi_{2 F}(\xi)+\left|m_{0}(\xi / 2)\right|^{2} \chi_{2 F-2 \pi}(\xi) .
$$

On the other hand, by Corollary 1.2 and Remark 1.3, we also have

$$
(2 \pi) \sum_{k \in \mathbb{Z}}|\hat{\psi}(\xi+2 k \pi)|^{2}=\chi_{E}(\xi)
$$

where $E=(2 F \cap 2(F-\pi)) \cup((2 F \Delta 2(F-\pi)) \backslash F)$. But from Lemma 3.1 and (3.5), we have that when $\xi \in(2 F \Delta 2(F-\pi)) \backslash F$,

$$
\sum_{k \in \mathbb{Z}}|\hat{\psi}(\xi+2 k \pi)|^{2}=0 .
$$

This implies that $(2 F \Delta 2(F-\pi)) \backslash F$ must have measure 0 . Hence $(2 F \Delta 2(F-\pi))$ $\subset F$ (modulo a null set). By Corollary 1.2, we also have $2 F \cap 2(F-\pi) \subset F$. So $2 F \cup(2 F-2 \pi) \subset F$ and so $2 F \subset F$. A standard Lebesgue point argument (cf. LMS, the proof of Lemma 2) shows that $\bigcup_{\ell \in \mathbb{Z}}(F+2 \ell \pi)=\mathbb{R}$ (modulo a null set). Thus $F=\mathbb{R}$ (modulo a null set) since $F$ is $2 \pi$-translation invariant.

Lemma 3.3. Suppose that $\left\{T_{\ell} \phi: \ell \in \mathbb{Z}^{d}\right\}$ is a normalized tight frame for a shiftinvariant subspace $M$. Let $\phi_{0} \in M$ be such that $\left\{T_{\ell} \phi_{0}: \ell \in \mathbb{Z}^{d}\right\}$ is a normalized tight frame for $\overline{\operatorname{span}}\left\{T_{\ell} \phi_{0}: \ell \in \mathbb{Z}^{d}\right\}$. Then $\left\{T_{\ell} \phi_{0}: \ell \in \mathbb{Z}^{d}\right\}$ is a normalized tight frame for $M$ if and only if

$$
\sum_{\ell \in \mathbb{Z}^{d}}|\phi(\xi+2 \ell \pi)|^{2}=\sum_{\ell \in \mathbb{Z}^{d}}\left|\phi_{0}(\xi+2 \ell \pi)\right|^{2}
$$

holds a.e.

Proof. Let $P$ be the orthogonal projection of $M$ onto $\overline{\operatorname{span}}\left\{T_{\ell} \phi_{0}: \ell \in \mathbb{Z}^{d}\right\}$. Then $\left\{T_{\ell} P \phi: \ell \in \mathbb{Z}^{d}\right\}$ is a normalized tight frame for $\overline{\operatorname{span}}\left\{T_{\ell} \phi_{0}: \ell \in \mathbb{Z}^{d}\right\}$. Thus, by Lemma 2.1, $\left\|\phi_{0}\right\|=\|P \phi\|$. However, by $\sum_{\ell \in \mathbb{Z}^{d}}|\phi(\xi+2 \ell \pi)|^{2}=\sum_{\ell \in \mathbb{Z}^{d}}\left|\phi_{0}(\xi+2 \ell \pi)\right|^{2}$, we also have $\|\phi\|=\left\|\phi_{0}\right\|$, which implies $\|P \phi\|=\|\phi\|$. Thus $\frac{\sum}{\operatorname{span}}\left\{T_{\ell} \phi_{0}: \ell \in \mathbb{Z}^{d}\right\}=$ $M$ since the $\phi$ translation generates $M$. 
Now assume that $\left(V_{j}\right)$ is an FMRA with a frame scaling function $\phi$, and assume that $m_{0}$ satisfies (3.1). Let $K_{1}=F \cap(F-\pi), K_{2}=(F \Delta(F-\pi)) \cap F / 2$ and $K_{3}=(F \Delta(F-\pi)) \backslash(F / 2)$. Then $K_{1}, K_{2}$ and $K_{3}$ are disjoint and $\pi$-translation stable. We define the $2 \pi$-periodic function $m_{1}$ by

$$
m_{1}(\xi)= \begin{cases}e^{i \xi} \overline{m_{0}(\xi+\pi)}, & \xi \in K_{1}, \\ 1, & \xi \in K_{3}, \\ 0, & \text { everywhere else, }\end{cases}
$$

and define $\psi$ by

$$
\hat{\psi}(\xi)=m_{1}(\xi / 2) \hat{\phi}(\xi / 2) .
$$

Theorem 3.4. Assume that $\left(V_{j}\right)$ is associated with a wavelet frame (equivalently, $2 F \cap 2(F-\pi) \subset F \subset 2 F \cup 2(F-\pi)$ by Corollary 1.2). Then $\psi$ defined by (3.4) is an FMRA wavelet frame.

Proof. By assumption there exists a function $f \in W_{0}$ such that $\left\{T_{\ell} f: \ell \in \mathbb{Z}\right\}$ is a normalized tight frame for $W_{0}$. Therefore, by Corollary 1.2, $\sum_{k \in \mathbb{Z}}|\hat{f}(\xi+2 k \pi)|^{2}=$ $\frac{1}{2 \pi} \chi_{E}$, where $E=2\left(K_{1} \cup K_{3}\right)$. By Lemma 3.3 , it suffices to prove that (i) $\psi \in W_{0}$ and (ii) $\sum_{k \in \mathbb{Z}}|\hat{\psi}(\xi+2 k \pi)|^{2}=\frac{1}{2 \pi} \chi_{E}$.

In order that $\psi \in W_{0}$, by Theorem 5.1 in [BL], we need to verify the following:

$$
\overline{m_{0}(\xi)} m_{1}(\xi) \chi_{F}(\xi)+\overline{m_{0}(\xi+\pi)} m_{1}(\xi+\pi) \chi_{F-\pi}=0 .
$$

Suppose that $\xi \in K_{1}$. Then $m_{1}(\xi)=e^{i \xi} \overline{m_{0}(\xi+\pi)}$ and $m_{1}(\xi+\pi)=-e^{i \xi} \overline{m_{0}(\xi)}$. Hence (3.5) holds in this case. If $\xi \in K_{2}$, then $\xi+\pi \in K_{2}$ (note that $F / 2$ is $\pi$-translation stable). Hence $m_{1}(\xi)=m_{1}(\xi+\pi)=0$, which implies (3.5) holds. If $\xi \in K_{3}$, then by Lemma $3.1\left|m_{0}(\xi)\right|^{2}+\left|m_{0}(\xi+\pi)\right|^{2}=0$, which also implies that (3.5) holds. Therefore we proved $(i)$.

Now we verify $(i i)$. From (3.4), we have

$$
(2 \pi) \sum_{k \in \mathbb{Z}}|\hat{\psi}(\xi+2 k \pi)|^{2}=\left|m_{1}(\xi / 2)\right|^{2} \chi_{2 F}(\xi)+\left|m_{1}(\xi / 2+\pi)\right|^{2} \chi_{2 F-2 \pi}(\xi) .
$$
Thus

By Lemma 3.1, $\left|m_{0}(\xi / 2)\right|^{2}+\left|m_{0}(\xi / 2+\pi)\right|^{2}=1$ when $\xi \in 2 F \cap 2(F-\pi)\left(=2 K_{1}\right)$.

$$
\begin{aligned}
(2 \pi) \sum_{k \in \mathbb{Z}}|\hat{\psi}(\xi+2 k \pi)|^{2} & =\left|m_{1}(\xi / 2)\right|^{2}+\left|m_{1}(\xi / 2+\pi)\right|^{2} \\
& =\left|m_{0}(\xi / 2+\pi)\right|^{2}+\left|m_{0}(\xi / 2)\right|^{2}=1 .
\end{aligned}
$$

If $\xi \in(2 F \Delta 2(F-\pi)) \backslash F\left(=2 K_{3}\right)$, then

$$
m_{1}(\xi / 2)=m_{1}(\xi / 2+\pi)=1
$$

by the definition of $m_{1}$. Hence

$$
(2 \pi) \sum_{k \in \mathbb{Z}}|\hat{\psi}(\xi+2 k \pi)|^{2}=\left|m_{1}(\xi / 2)\right|^{2} \chi_{2 F}(\xi)+\left|m_{1}(\xi / 2+\pi)\right|^{2} \chi_{2 F-2 \pi}(\xi)=1 .
$$

Similarly

$$
(2 \pi) \sum_{k \in \mathbb{Z}}|\hat{\psi}(\xi+2 k \pi)|^{2}=\left|m_{1}(\xi / 2)\right|^{2} \chi_{2 F}(\xi)+\left|m_{1}(\xi / 2+\pi)\right|^{2} \chi_{2 F-2 \pi}(\xi)=0
$$


when $\xi \notin E$, since $\chi_{2 F}(\xi)=\chi_{2 F-2 \pi}(\xi)=0$ if $\xi \notin 2 F \cup 2(F-\pi)$, and $m_{1}(\xi / 2)=$ $m_{1}(\xi / 2+\pi)=0$ if $\xi \in 2 K_{2}$. Considering all the cases, we obtain

$$
\sum_{k \in \mathbb{Z}}|\hat{\psi}(\xi+2 k \pi)|^{2}=\frac{1}{2 \pi} \chi_{E},
$$

as claimed.

Remark. Since this paper was submitted, a more general result on the construction of wavelet frames from filters and frame scaling functions for an FMRA has been proved by Baggett, Courter and Merrill (Corollary 3.5, [BCM]). The result in $[\mathrm{BCM}$ deals with general dilation matrices. However, it is evident that our construction formula (3.4) (for the special dilation $d=2$ ) is more explicit and may be more accessible than the general result.

\section{ACKNOWLEDGEMENT}

The authors thank the referee for several valuable suggestions, especially for pointing out the connection between the results in this paper and the results in [RS2] and [BCM].

\section{REFERENCES}

[Aus] P. Auscher, Solution of two problems on wavelets, J. Geometric Analysis, 5 (1995), 181-236. MR.1341029 (96g:42016)

[Ba] L. Baggett, An abstract interpretation of the wavelet dimension function using group representations, J. Funct. Anal., 173 (2000), 1-20. MF1760275 (2001j:42028)

$[\mathrm{BCM}]$ L. Baggett, J. Courter and K. Merrill, The construction of wavelets from generalized conjugate mirror filters in $L^{2}\left(\mathbb{R}^{n}\right)$, Appl. Comput. Harmonic Anal., 3 (2002), 201-223. MR1942742 (2004d:42054)

[BMM] L. Baggett, H. Medina and K. Merrill, Generalized multiresolution analysis, and a construction procedure for all wavelet sets in $\mathbb{R}^{n}$, J. Fourier Analysis and Applications, 5 (1999), 563-573. MR.1752590 (2001f:42055)

[BM] L. Baggett and K. Merrill, Abstract harmonic analysis and wavelets in $\mathbb{R}^{n}$, Contemp. Math., 247 (1999), 17-27. MR1735967 (2001b:42043)

[BL] J. Benedetto and S. Li, The theory of multiresolution analysis frames and applications to filter banks, Appl. Comput. Harmon. Anal., 5(1998), 389-427. MF 1646534 (99k:42054)

[Bo] M. Bownik, The structure of shift-invariant subspaces of $L^{2}\left(\mathbb{R}^{n}\right)$, J. Functional Analysis, 177 (2000), 282-309. MR1795633 (2001k:42037)

[BRS] M. Bownik, Z. Rzeszotnik and D. Speegle, A characterization of dimension functions of wavelets, Appl. Comput. Harmon. Anal., 10 (2001), 71-92. MR1808201 (2001m:42058)

[Cou] J.Courter, Construction of dilation-d wavelets, Contemp. Math., 247 (1999), 183-205. MR 1738090 (2001b:42048)

[DDR] C. deBoor, R. DeVore and A. Ron, The structure of finitely generated shift-invariant spaces in $L^{2}\left(\mathbb{R}^{d}\right)$, J. Funct. Anal., 119 (1994), 37-78. MR.1255273 (95g:46050)

[GHa] J.-P. Gabardo and D. Han, Subspace Weyl-Heisenberg frames, J. Fourier Analysis and Applications, 7(2001), 419-433. MR 1836821 (2002f:42031)

[Le1] P.-G. Lemarié-Rieusset, Existence de "fonction-pére" pour les ondelettes à support compact, C. R. Acad. Sci. Paris Sér. I Math 314(1992), 17-19. MR1149631 (93c:42033)

[Le2] P.-G. Lemarié-Rieusset, Sur l'existence des analyses multi-résolutions en théorie des ondelettes, Rev. Mat. Iberoamericana, 8(1992), 457-474. MR 1202418 (94g:42058)

[LMS] R. Lorentz, W. Madych and A. Sahakian Translation and dilation invariant subspaces of $L^{2}(\mathbb{R})$ and multiresolution analyses, Appl. Comp. Harm. Anal., 5 (1998), 375-388. MR1646510 (99h:42062)

[Ma] S. Mallat, Multiresolution approximations and wavelet orthonormal basis of $L^{2}(\mathbb{R})$, Trans. Amer. Math. Soc., 315 (1989) 69-87. MR1008470 (90e:42046) 
[Pa] M. Papadakis, On the dimension function of orthonormal wavelets, Proc. Amer. Math. soc., 128 (2000), 2043-2049. MR.1654108 (2000m:42031)

[RS1] A. Ron and Z. Shen, Frames and stable bases for shift-invariant subspaces of $L^{2}\left(\mathbb{R}^{d}\right)$, Canadian J. Math., 7 (1995), 1051-1094. MR1350650 (96k:42049)

[RS2] A. Ron and Z. Shen, The wavelet dimension function is the trace function of a shift-invariant system Proc. Amer. Math. Soc., 131 (2003), 1385-1398. MR1949868 (2003i:42055)

[We] E. Weber, Applications of the wavelet multiplicity function, Contemp. Math., Amer. Math. Soc., Providence, RI, 247(1999), 297-306. MR1738096 (2001f:42064)

Department of Mathematics, East China Normal University, Shanghai, Peoples RePUBLIC OF CHINA

Department of Mathematics, University of Central Florida, Orlando, Florida 32816

E-mail address: dhan@pegasus.cc.ucf.edu 